\title{
Prognostic factors in 868 advanced gastric cancer patients treated with second-line chemotherapy in the real world
}

\author{
Valentina Fanotto ${ }^{1} \cdot$ Stefano Cordio $^{2} \cdot$ Giulia Pasquini $^{3} \cdot$ Caterina Fontanella $^{1}$. \\ Lorenza Rimassa $^{4}$ - Francesco Leone ${ }^{5}$ - Gerardo Rosati ${ }^{6}$ - Daniele Santini ${ }^{7}$. \\ Riccardo Giampieri $^{8}$ - Samantha Di Donato ${ }^{9}$ - Gianluca Tomasello ${ }^{10}$. \\ Nicola Silvestris ${ }^{11} \cdot$ Filippo Pietrantonio $^{12}$ - Francesca Battaglin $^{13}$. \\ Antonio Avallone $^{14}$ - Mario Scartozzi ${ }^{15}$. Eufemia Stefania Lutrino ${ }^{16}$. \\ Davide Melisi $^{17} \cdot$ Lorenzo Antonuzzo $^{18} \cdot$ Antonio Pellegrino $^{19} \cdot$ Valter Torri $^{20}$. \\ Giuseppe Aprile ${ }^{1}$
}

Received: 31 August 2016/Accepted: 8 December 2016/Published online: 27 December 2016

(C) The International Gastric Cancer Association and The Japanese Gastric Cancer Association 2016

\begin{abstract}
Background Although second-line therapy is often considered for advanced gastric cancer patients, the optimal candidates are not well defined.

Methods We retrospectively collected baseline parameters, tumour features, and treatment data for 868 advanced gastric cancer patients exposed to multiple treatment lines at 19 Italian centres. Cross-tables and chi-square tests were
\end{abstract}

Electronic supplementary material The online version of this article (doi:10.1007/s10120-016-0681-6) contains supplementary material, which is available to authorized users.

Giuseppe Aprile

giuseppe.aprile@asuiud.sanita.fvg.it

1 Dipartimento di Oncologia, Azienda OspedalieroUniversitaria "Santa Maria della Misericordia" Piazzale Santa Maria della Misericordia 15, 33100 Udine, Italy

2 Department of Oncology, Garibaldi Nesima Hospital, Catania, Italy

3 UO Oncologia Medica 2 Universitaria, Ospedale S. ChiaraAzienda Ospedaliero-Universitaria Pisana Istituto Toscano Tumori, Pisa, Italy

4 Medical Oncology and Hematology Unit, Humanitas Cancer Center, Humanitas Clinical and Research Center, Rozzano, MI, Italy

5 Institute for Cancer Research and Treatment IRCCS, Candiolo, TO, Italy

6 Medical Oncology, San Carlo Hospital, Potenza, Italy

7 Medical Oncology, Campus Bio-Medico University, Rome, Italy

8 Medical Oncology, Ospedali Riuniti, Ancona, Italy

9 Department of Medical Oncology, General Hospital, Prato, Italy used to describe categorical features. To predict the impact of clinical variables on progression-free survival and overall survival, Kaplan-Meier and Cox regression analyses were performed.

Results At the start of second-line therapy, median age was 64.8 years (25th-75th percentiles: 55.2-71.9 years). Overall, $43 \%$ of patients received single-agent chemotherapy, $47.4 \%$ a doublet, and $7.3 \%$ a triplet. Median second-line progression-free survival was 2.8 months (25th-75th percentiles: $1.8-5.2$ months) and median second-line overall survival was 5.6 months (25th-75th

10 Medical Oncology Division, Istituti Ospitalieri di Cremona, Cremona, Italy

11 Medical Oncology Unit, National Cancer Institute IRCCS "Giovanni Paolo II", Bari, Italy

12 Medical Oncology, National Cancer Institute IRCCS, Milan, Italy

13 UOC Oncologia Medica 1, Dipartimento di Oncologia Clinica e Sperimentale, Istituto Oncologico Veneto-IRCCS, Padua, Italy

14 Gastrointestinal Oncology, National Cancer Institute, IRCCS "Fondazione G. Pascale", Naples, Italy

15 Medical Oncology, University Hospital, University of Cagliari, Cagliari, Italy

16 Medical Oncology, Perrino Hospital, Brindisi, Italy

17 Medical Oncology, University of Verona, Verona, Italy

18 Medical Oncology, Careggi University Hospital, Florence, Italy

19 Medical Oncology, Vito Fazzi Hospital, Lecce, Italy

20 Oncology Unit, IRCCS Mario Negri Institute, Milan, Italy 
percentiles: 2.9-10.0 months). Multivariate analysis showed that performance status, LDH level, neutrophils/lymphocytes ratio, and progression-free survival in the first-line therapy all impacted on prognosis. Based on these four prognostic factors, a prognostic index was constructed that divided patients into good, intermediate, and poor risk groups; median second-line overall survival for each group was 7.7, 4.5, and 2.0 months, respectively (logrank $p<0.0001)$.

Conclusions Advanced gastric cancer patients with a favourable ECOG performance status, lower LDH levels, and a lower neutrophils/lymphocytes ratio at the start of second-line therapy seem to have better outcomes, regardless of age and intensity of treatment. A longer progression-free survival in the first-line therapy also had positive prognostic value. Our real-life study might help clinicians to identify the patients who may benefit most from a second-line therapy.

Keywords Advanced gastric cancer - Second-line chemotherapy - Prognostic factors - Overall survival · Progression-free survival

\section{Introduction}

Gastric cancer is the fourth most common malignant tumour worldwide and the second leading cause of cancerrelated mortality globally, accounting for 841,000 deaths in 2013 [1]. It is more common in men and it is more frequently diagnosed among people aged 65-74 years. The number of new cases and death rates have decreased over the last decade; nevertheless, the prognosis for patients with metastatic disease remains poor, with a 5-year relative survival of around 5\% [2]. Despite radical surgical resection, less than $30 \%$ of patients with localised gastric cancer are eventually cured, and palliative chemotherapy is commonly used to prolong survival, improve symptoms, and ameliorate the quality of life (QoL) of patients with metastatic disease [3]. Since combination regimens are associated with higher response rates and increased survival compared with single-agent chemotherapies [4], the first-line standard of care encompasses a combination of fluoropyrimidine and a platinum-containing regimen, while the use of a triplet including an anthracycline or a taxane is currently restricted to locally advanced disease or carefully selected patients with distant metastases [5, 6]; in the human epidermal growth factor receptor (HER)-2 enriched population, trastuzumab is added to a platinum-based chemotherapy [7]. Many patients, however, present a primary refractory disease or eventually progress [4], and their median overall survival only occasionally surpasses 12 months. The role of second-line therapy for metastatic gastric cancer has recently been the focus of debate [8, 9], at least in Western countries. Indeed, the proportion of patients offered second-line therapy ranges from $14 \%$ of those enrolled in Western clinical trials (e.g. the REAL-2 trial) [5] to $75 \%$ of patients participating in Asian studies (e.g. the SPIRITS trial) [10]. Recently, a number of clinical trials have clearly defined the role of second-line treatment in improving patients' survival and QoL [11-14]. How to select optimal candidates for second-line chemotherapy in clinical practice is, however, still unclear, and many factors may influence the choice: patient's performance status (PS), previously experienced toxicity, burden of disease, and availability of non-cross-resistant drugs. Several efforts have been made to define the patients that will derive the largest benefit from a first- and second-line chemotherapeutic regimen. Nevertheless, the search for predictive factors or validated prognostic indices that can be used to define who may profit most from second-line therapy is still ongoing. We aimed to explore which factors could help clinicians to individuate patients who deserve further oncologic treatment beyond disease progression to first-line chemotherapy.

\section{Patients and methods}

This is a multicentre, retrospective report involving 19 Italian oncologic departments; the study covered a large part of the country, since six centres were located in the north, six in Central Italy, and seven in the south. Medical records of approximately 2200 advanced gastric cancer (AGC) patients were reviewed, which led to the selection of 868 eligible cases that received at least two lines of systemic treatment from January 2006 to October 2015.

All the patients had a histologically confirmed diagnosis of metastatic gastric carcinoma. The inclusion criteria for the study included progressive disease after first-line chemotherapy and having received at least 1 cycle of a second-line therapy for AGC. Baseline parameters, tumour characteristics, and treatment data were all reviewed and anonymously collected. Laboratory variables were initially recorded as continuous variables and later dichotomised according to the median value of each variable. PS was evaluated according to the Eastern Cooperative Oncology Group (ECOG) criteria. For each patient included, we registered ECOG PS, haemoglobin level, platelet (PLT) count, lactate dehydrogenase (LDH) level, and neutrophils/lymphocytes ratio at the initiation of second-line chemotherapy. All the data were handled in anonymised format. Predefined endpoints of the observational study were the response rate (according to the investigators' assessment), overall survival (OS) in second-line therapy (which was calculated as the time interval between the beginning of second-line chemotherapy and the date of 
death or the last follow-up visit), and progression-free survival (PFS) in second-line therapy (which was measured from the start of second-line chemotherapy to the date of evidence of progressive disease, death, or the last followup visit-whichever came first). PFS and OS were estimated using the Kaplan-Meier method, with 95\% HallWeller bands and log rank. Cox regression analyses were used to calculate hazard ratios (HR) and 95\% confidence interval $(95 \% \mathrm{CI})$. Factors included in the univariate analyses were age, intensity of second-line chemotherapy (doublet/triplet vs. single-agent chemotherapy), progression-free survival achieved in first-line therapy (PFS in first line $\geq 6.8$ vs. $<6.8$ months, median PFS in first line 6.9 months, 25 th-75th percentiles: $4.3-10.2$ months), LDH level ( $\leq 480$ vs. $>480 \mathrm{UI} / \mathrm{L} ; 480 \mathrm{UI} / \mathrm{L}$ represents the upper limit of normal LDH level reported in certified Italian laboratories), PLT count $\left(<450,000 / \mathrm{mm}^{3}\right.$ vs. $\geq 450,000 /$ $\mathrm{mm}^{3} ; 450,000 / \mathrm{mm}^{3}$ represents the upper limit of normal PLT count), ECOG PS ( 0 vs. $2-3$ and 1 vs. $2-3$ ), and neutrophils/lymphocytes ratio $(<2.7$ vs. $\geq 2.7 ; 2.7$ represents the median value of neutrophils/lymphocytes ratio in our series; a similar value was reported in the literature). PFS in first-line therapy was measured from the start of first-line chemotherapy to the date of evidence of progressive disease; the definition of first-line chemotherapy was also applied to those patients who recurred within 6 months from surgery after neoadjuvant chemotherapy and to those patients who recurred within 6 months from the end of adjuvant chemotherapy. Cross-tables and the chi-square test were used to describe categorical features. To predict the impact of clinical variables on PFS and OS, we performed Kaplan-Meier analysis, and Cox regression analysis was used to estimate crude and adjusted HR with 95\% CIs. For the score index estimation, the Cox model was used with a backward stepwise entry procedure. This approach allows for the removal of one variable at a time until no reduction in predictive power is found [this is a more robust method than forward entry, and limits the possibility of overfitting the data]. A statistical significance cutoff of $p=0.05$ was used to retain the variables in the final model. Once the final model had been obtained, the coefficient estimates were "normalised" by dividing by the smallest one and rounding the resulting ratios to the nearest integer value.

Score-value grouping was then performed by applying the nonparametric tree modelling technique of classification and regression tree analysis (CART). The area under the receiver characteristic curve (AUC) method was used to quantify the accuracy of the model. Statistical analysis was performed using the $\mathrm{SAS}^{\odot}$ statistical package 9.2 (SAS Institute Inc., Cary, NC, USA). The R software was also used for CART analysis.

\section{Results}

Data from 868 AGC patients were entered from the 19 participating sites and included in the analysis. Complete clinical and pathologic characteristics of the patients are shown in Table 1 . The median patient age at diagnosis of stage IV was 64 years (25th-75th percentiles: 54.5-71.3 years), whereas the median age at the start of second-line treatment was 64.8 years (25th-75th percentiles: $55.2-71.9$ years). 587 patients $(67.6 \%)$ were male. ECOG PS at the initiation of the second-line therapy was 0 or 1 in $702(80.9 \%)$ patients. As expected, the most common site of the primary tumour was the gastric body $(30.5 \%)$. The intestinal histotype accounted for $41.5 \%$ of cases, $50.8 \%$ of patients had poorly differentiated or undifferentiated tumours, and $12.4 \%$ presented HER2-positive disease. Stage IV disease was diagnosed at initial presentation (de novo disease) in 549 (63.2\%) patients. $384(44.2 \%)$ patients had multiple metastatic sites at the time of diagnosis of metastatic disease. Types of treatment received are listed in Table 2. A previous surgical treatment (of any type) was reported in 458 cases (52.8\%): total gastrectomy in 222, partial gastrectomy in 197, and other palliative intervention in 39 cases. When performed, lymphadenectomy was most frequently D2 or D3 in extent. In the first-line setting, most of the patients received a combination regimen (90.2\%); fluoropyrimidines were the most frequently used agents (92.4\%), followed by platinum salts $(81.5 \%)$, anthracyclines (22.6\%), and taxanes (16.8\%). In detail, the most frequently administered combination regimens (doublet or triplet chemotherapy) in the first-line therapy were: oxaliplatin plus a fluoropyrimidine (oral or intravenous) in $23.02 \%$ (195/847) of cases, cisplatin plus a fluoropyrimidine (oral or intravenous) in $16.06 \%$ (136/847) of cases, and epirubicin plus cisplatin plus a fluoropyrimidine (oral or intravenous) in $12.75 \%$ (108/847) of cases. In $13.8 \%$ of cases, patients received a biological agent in the first-line therapy (a small number of them were participating in a clinical trial). A complete response to the first-line chemotherapy was achieved in 27 patients and a partial response in 273 patients, for an overall response rate of $34.6 \%$. Most of the patients received a combination regimen in the second-line therapy too $(54.7 \%)$, but the percentage of those who received single-agent chemotherapy was significantly increased (43.0 vs. $7.4 \%$ ). Most patients received either a fluoropyrimidine-based or an irinotecan-based chemotherapy (50.1 and 42.6\%, respectively). In detail, of 195 patients treated in the firstline therapy with oxaliplatin plus a fluoropyrimidine, 66 received FOLFIRI in the second line $(33.85 \%), 64$ docetaxel $(32.82 \%)$, and 14 paclitaxel $(7.18 \%)$. Of 136 
Table 1 Patients' clinical and pathologic characteristics

\begin{tabular}{|c|c|}
\hline Characteristic & Number of patients $(\%)$ \\
\hline \multicolumn{2}{|l|}{ Gender } \\
\hline Male & $587(67.6)$ \\
\hline Female & $281(32.4)$ \\
\hline \multicolumn{2}{|c|}{ ECOG PS at start of second-line chemotherapy } \\
\hline 0 & $259(29.8)$ \\
\hline 1 & $443(51.0)$ \\
\hline $2-3$ & $157(18.1)$ \\
\hline Missing & $9(1.1)$ \\
\hline \multicolumn{2}{|l|}{ Site of primary tumour } \\
\hline Gastric body & $265(30.5)$ \\
\hline Antropylorus & $168(19.4)$ \\
\hline Cardia & $165(19.0)$ \\
\hline Fundus & $120(13.8)$ \\
\hline Gastro-oesophageal junction & $118(13.6)$ \\
\hline Missing & $32(3.7)$ \\
\hline \multicolumn{2}{|l|}{ Histotype } \\
\hline Intestinal & $360(41.5)$ \\
\hline Diffuse & $230(26.5)$ \\
\hline Other & $17(2.0)$ \\
\hline Unknown & $261(30.0)$ \\
\hline \multicolumn{2}{|l|}{ Grade of differentiation } \\
\hline Well-moderate & $221(25.5)$ \\
\hline Poor-undifferentiated & $441(50.8)$ \\
\hline Missing & $206(23.7)$ \\
\hline \multicolumn{2}{|l|}{ HER-2 status } \\
\hline Negative & $481(55.4)$ \\
\hline Positive & $108(12.4)$ \\
\hline Missing & $279(32.2)$ \\
\hline \multicolumn{2}{|l|}{ Stage at diagnosis } \\
\hline I & $10(1.2)$ \\
\hline II & $56(6.5)$ \\
\hline III & $240(27.6)$ \\
\hline IV & $549(63.2)$ \\
\hline Missing & $13(1.5)$ \\
\hline \multicolumn{2}{|c|}{ Number of metastatic sites at stage IV diagnosis } \\
\hline 1 & $475(54.7)$ \\
\hline 2 & $273(31.5)$ \\
\hline$\geq 3$ & $111(12.8)$ \\
\hline Missing & $9(1.0)$ \\
\hline \multicolumn{2}{|c|}{ Locations of metastases at stage IV diagnosis } \\
\hline Lymph nodes & $375(43.2)$ \\
\hline Liver & $330(38.0)$ \\
\hline Peritoneum or ovary & $311(35.8)$ \\
\hline Lung & $78(9.0)$ \\
\hline Other & $127(14.6)$ \\
\hline
\end{tabular}

ECOG Eastern Cooperative Oncology Group, PS performance status, $H E R$ human epidermal growth factor receptor
Table 2 Types of treatments received

\begin{tabular}{|c|c|}
\hline Treatment & Number of patients $(\%)$ \\
\hline \multicolumn{2}{|l|}{ Surgery } \\
\hline Total gastrectomy & $222(25.6)$ \\
\hline Partial gastrectomy & $197(22.7)$ \\
\hline Palliative intervention & $39(4.5)$ \\
\hline Lymphadenectomy & $402(46.3)$ \\
\hline D1 lymphadenectomy & $21(2.4)$ \\
\hline D2 or D3 lymphadenectomy & $381(43.9)$ \\
\hline \multicolumn{2}{|l|}{ First-line chemotherapy } \\
\hline \multicolumn{2}{|l|}{ Intensity of treatment } \\
\hline Single-agent chemotherapy & $64(7.4)$ \\
\hline Doublet chemotherapy & $433(49.9)$ \\
\hline Triplet chemotherapy & $350(40.3)$ \\
\hline Missing & $21(2.4)$ \\
\hline \multicolumn{2}{|l|}{ Type of agent used } \\
\hline Fluoropyrimidines & $802(92.4)$ \\
\hline Platinum salts & 707 (81.5) \\
\hline Anthracyclines & 196 (22.6) \\
\hline Taxanes & $146(16.8)$ \\
\hline Biologics & $120(13.8)$ \\
\hline Irinotecan & $90(10.4)$ \\
\hline \multicolumn{2}{|l|}{ Second-line chemotherapy } \\
\hline \multicolumn{2}{|l|}{ Intensity of treatment } \\
\hline Single-agent chemotherapy & $373(43.0)$ \\
\hline Doublet chemotherapy & $411(47.4)$ \\
\hline Triplet chemotherapy & $63(7.3)$ \\
\hline Chemo-free therapy & $21(2.3)$ \\
\hline \multicolumn{2}{|l|}{ Type of agents used } \\
\hline Fluoropyrimidines & $435(50.1)$ \\
\hline Irinotecan & $370(42.6)$ \\
\hline Taxanes & $351(40.4)$ \\
\hline Platinum salts & $130(15.0)$ \\
\hline Anthracyclines & $42(4.8)$ \\
\hline Biologics & $42(4.8)$ \\
\hline
\end{tabular}

patients treated in the first line with cisplatin plus a fluoropyrimidine, 56 received FOLFIRI in the second line (41.18\%), 31 docetaxel (22.79\%), and 20 paclitaxel $(14.71 \%)$. Of 108 patients treated in the first line with epirubicin plus cisplatin plus a fluoropyrimidine, 41 received docetaxel in the second line $(37.96 \%), 20$ FOLFIRI/XELIRI (18.52\%), and 13 paclitaxel (12.04\%). Of 435 patients who received a fluoropyrimidine in the second line, $383(88 \%)$ received 5-fluorouracil while 52 (12\%) received capecitabine. Of 349 patients who received a taxane in the second line, 261 of them (74.8\%) received docetaxel while $88(25.2 \%)$ received paclitaxel. Only $4.8 \%$ of patients received a biological drug in the 
second line. In particular, 31 out of 868 patients (3.6\%) received ramucirumab as second-line therapy, 17 as a single agent and 14 in combination with paclitaxel. A complete response to second-line chemotherapy was achieved in 12 patients and a partial response in 79 patients, for an overall response rate of $10.5 \%$. In terms of treatment duration, the median number of cycles received was six in the first line (around 4 months) and four in the second line (around 3 months). 331 out of 868 patients received a third-line therapy too.

At the time of analysis, $813(93.7 \%)$ patients had died, mainly because of tumour progression. Median survival from the diagnosis of stage IV for the whole cohort was 13.1 months (25th-75th percentiles: 8.9-19.7 months). Median PFS in the first-line treatment was 6.9 months (25th75th percentiles: $4.3-10.2$ months), whereas median PFS in the second-line treatment was 2.8 months (25th-75th percentiles: 1.8-5.2 months). Median OS in the second line was 5.6 months (25th-75th percentiles: 2.9-10.0 months).
At univariate analysis (Table 3), six variables were significantly associated with longer survival in the second line (PFS and OS): ECOG PS 0 or 1, PLT count $<450,000 /$ $\mathrm{mm}^{3}$, LDH level $\leq 480 \mathrm{UI} / \mathrm{L}$, intensity of treatment (doublet or triplet vs. single-agent chemotherapy), neutrophils/lymphocytes ratio $<2.7$, and having achieved a PFS in the first-line therapy $\geq 6.8$ months. On the contrary, younger age was linked to a poor survival outcome. Patients younger than 40 years experienced inferior PFS and OS in the second line compared to those older than 40 (median PFS in the second line: 1.9 vs. 2.9 months, HR 1.91, 95\% CI 1.33-2.74, $p<0.0001$; median OS in the second line: 3.9 vs. 5.8 months, HR 1.84, 95\% CI $1.28-2.64, p=0.001)$.

Although the number of very old patients was limited (122 patients, $14.1 \%$ ), no statistically significant survival difference was found between patients who were younger or older than 75 years in either PFS or OS, suggesting that age per se should not limit the use of second-line treatment.

Table 3 Univariate analysis

\begin{tabular}{|c|c|c|c|c|c|c|c|c|}
\hline \multirow[t]{2}{*}{ Variable } & \multicolumn{4}{|c|}{ PFS in second-line therapy } & \multicolumn{4}{|c|}{ OS in second-line therapy } \\
\hline & Median (months) & HR & $95 \% \mathrm{CI}$ & $p$ & Median (months) & HR & $95 \%$ CI & $p$ \\
\hline \multicolumn{9}{|l|}{ ECOG PS } \\
\hline 0 vs. $2-3$ & 3.9 vs. 1.7 & 0.41 & $0.33-0.50$ & $<0.0001$ & 8.7 vs. 2.6 & 0.30 & $0.25-0.37$ & $<0.0001$ \\
\hline 1 vs. $2-3$ & 2.9 vs. 1.7 & 0.54 & $0.45-0.65$ & $<0.0001$ & 5.5 vs. 2.6 & 0.49 & $0.41-0.59$ & $<0.0001$ \\
\hline \multicolumn{9}{|l|}{ PLT count } \\
\hline$<450,000 / \mathrm{mm}^{3}$ vs. & 2.8 & 0.72 & $0.53-0.97$ & 0.029 & 5.6 & 0.63 & $0.47-0.85$ & 0.002 \\
\hline$\geq 450,000 / \mathrm{mm}^{3}$ & 2.2 & & & & 4.2 & & & \\
\hline \multicolumn{9}{|l|}{ LDH level } \\
\hline \multirow[t]{2}{*}{$\leq 480 \mathrm{UI} / \mathrm{L}$ vs. $>480 \mathrm{UI} / \mathrm{L}$} & 2.8 & 0.56 & $0.44-0.71$ & $<0.0001$ & 5.9 & 0.56 & $0.44-0.71$ & $<0.0001$ \\
\hline & 2.0 & & & & 3.2 & & & \\
\hline \multicolumn{9}{|l|}{ Intensity of treatment } \\
\hline Doublet/triplet vs. & 3.2 & 0.77 & $0.67-0.88$ & $<0.0001$ & 6.5 & 0.81 & $0.70-0.93$ & 0.004 \\
\hline Single-agent CT & 2.5 & & & & 4.5 & & & \\
\hline \multicolumn{9}{|l|}{ Neutrophils/lymphocytes ratio } \\
\hline$<2.7$ vs. & 3.2 & 0.73 & $0.61-0.86$ & $<0.0001$ & 6.9 & 0.65 & $0.55-0.78$ & $<0.0001$ \\
\hline$\geq 2.7$ & 2.4 & & & & 4.1 & & & \\
\hline \multicolumn{9}{|l|}{ PFS in first-line therapy } \\
\hline$\geq 6.8$ months vs. & 3.2 & 0.75 & $0.66-0.86$ & $<0.0001$ & 7.0 & 0.67 & $0.58-0.77$ & $<0.0001$ \\
\hline$<6.8$ months & 2.6 & & & & 4.5 & & & \\
\hline \multicolumn{9}{|l|}{ Age } \\
\hline$<40$ years vs. & 1.9 & 1.91 & $1.33-2.74$ & $<0.0001$ & 3.9 & 1.84 & $1.28-2.64$ & 0.001 \\
\hline$\geq 40$ years & 2.9 & & & & 5.8 & & & \\
\hline \multicolumn{9}{|l|}{ Age } \\
\hline$\geq 75$ years vs. & 3.0 & 0.88 & $0.72-1.07$ & 0.188 & 6.9 & 0.84 & $0.69-1.03$ & 0.088 \\
\hline$<75$ years & 2.8 & & & & 5.6 & & & \\
\hline
\end{tabular}

ECOG Eastern Cooperative Oncology Group, $P S$ performance status, PLT platelets, $L D H$ lactate dehydrogenase, $C T$ chemotherapy, $P F S$ progression-free survival, $O S$ overall survival, $H R$ hazard ratio, $C I$ confidence interval 
Multivariate regression analysis (shown in Table 4) included the seven variables that were found to have prognostic significance in univariate analysis. Four of these factors, namely good ECOG PS, low LDH levels, low neutrophils/lymphocytes ratio, and longer PFS achieved in first-line therapy were independently associated with longer survival. Based on these four prognostic factors, a prognostic index was constructed that divided patients into good, intermediate, and poor risk groups. In multivariate analysis, ECOG PS 0 at the start of second-line therapy had a lower HR than ECOG PS 1, LDH level $\leq 480 \mathrm{UI} / \mathrm{L}$, neutrophil/lymphocytes ratio $<2.7$, and PFS in first-line therapy $\geq 6.8$ months. Based on the data obtained, each independent prognostic factor was assigned an index score depending on its degree of influence on the second-line OS: ECOG PS 0 at the start of the second-line therapy was assigned 4 points, ECOG PS 1 at the start of the secondline therapy was assigned 2 points, LDH level $\leq 480 \mathrm{UI} / \mathrm{L}$ at the start of the second-line therapy was assigned 1 point, neutrophils/lymphocytes ratio $<2.7$ at the start of the second-line therapy was assigned 1 point, and PFS in first-line therapy $\geq 6.8$ months was also assigned 1 point. ECOG PS $\geq 2$, LDH level $>480 \mathrm{UI} / \mathrm{L}$, neutrophils/lymphocytes ratio $\geq 2.7$, and PFS in first-line therapy $<6.8$ months were all assigned 0 points.

Further, a prognostic index which could range from 0 to 7 was developed for 430 out of the 868 patients for whom ECOG PS, LDH level, neutrophils/lymphocytes ratio at start of second-line therapy, and PFS in first-line therapy were all available.

Patients with a prognostic index of 5,6 , or 7 were categorised as the good risk group ( $n=193$; group 3 ), those with a prognostic index of 3 or 4 were categorised as the intermediate risk group ( $n=157$; group 2 ), and those with a prognostic index $\leq 2$ were categorised as the poor risk group ( $n=80$; group 1). Compared with group 1 , better outcomes were observed for group 2 (HR 0.492, 95\% CI $0.373-0.647, p<0.0001$ ) and group 3 (HR $0.257,95 \% \mathrm{CI}$ $0.195-0.339, p<0.0001)$; the median second-line OS for each group was 2.0 months (95\% CI 1.5-2.4), 4.5 months (95\% CI 3.7-5.2 months), and 7.7 months (95\% CI 6.9-8.7 months), respectively (log-rank $p<0.0001$ ).

\section{Discussion}

Despite improvements in our knowledge of AGC biology and the availability of novel treatment regimens which have led to significant improvements in PFS and OS in the first-line setting, the prognosis of AGC patients remains disappointingly poor. Second-line therapy is routinely used in many AGC patients, and different treatment strategies are currently available in clinical practice. Our study aimed to identify prognostic factors for survival in AGC patients exposed to a second-line therapy. The search for the optimal candidate for a first- and second-line chemotherapy for

Table 4 Multivariate analysis

\begin{tabular}{|c|c|c|c|c|c|c|}
\hline \multirow[t]{2}{*}{ Variable } & \multicolumn{3}{|c|}{ PFS in second-line therapy } & \multicolumn{3}{|c|}{ OS in second-line therapy } \\
\hline & $p$ & HR & $95 \% \mathrm{CI}$ & $p$ & HR & $95 \% \mathrm{CI}$ \\
\hline \multicolumn{7}{|l|}{ ECOG PS } \\
\hline 0 vs. $2-3$ & $<0.0001$ & 0.33 & $0.25-0.44$ & $<0.0001$ & 0.28 & $0.20-0.37$ \\
\hline \multicolumn{7}{|l|}{ ECOG PS } \\
\hline 1 vs. $2-3$ & $<0.0001$ & 0.57 & $0.44-0.75$ & $<0.0001$ & 0.53 & $0.40-0.69$ \\
\hline \multicolumn{7}{|l|}{ LDH level } \\
\hline$\leq 480$ vs. $>480 \mathrm{UI} / \mathrm{L}$ & 0.01 & 0.64 & $0.46-0.90$ & 0.037 & 0.70 & $0.50-0.98$ \\
\hline \multicolumn{7}{|l|}{ Neutrophils/lymphocytes ratio } \\
\hline$<2.7$ vs. $\geq 2.7$ & 0.007 & 0.75 & $0.61-0.93$ & $<0.0001$ & 0.66 & $0.53-0.81$ \\
\hline \multicolumn{7}{|l|}{ PFS in first-line therapy } \\
\hline$\geq 6.8$ vs. $<6.8$ months & 0.02 & 0.79 & $0.65-0.96$ & 0.002 & 0.72 & $0.59-0.89$ \\
\hline \multicolumn{7}{|l|}{ Age } \\
\hline$<40$ vs. $\geq 40$ years & 0.119 & 1.52 & $0.90-2.56$ & 0.065 & 1.63 & $0.97-2.74$ \\
\hline \multicolumn{7}{|l|}{ Intensity of treatment } \\
\hline Doublet/triplet vs. single-agent CT & 0.865 & 1.02 & $0.83-1.25$ & 0.672 & 1.05 & $0.85-1.29$ \\
\hline \multicolumn{7}{|l|}{ PTL count } \\
\hline$<450$ vs. $\geq 450 \times 10^{3} / \mathrm{mm}^{3}$ & 0.242 & 0.77 & $0.49-1.20$ & 0.406 & 0.83 & $0.53-1.29$ \\
\hline
\end{tabular}

ECOG Eastern Cooperative Oncology Group, $P S$ performance status, $L D H$ lactate dehydrogenase, $P F S$ progression-free survival, $C T$ chemotherapy, $P L T$ platelets, $O S$ overall survival, $H R$ hazard ratio, $C I$ confidence interval 
AGC has already been the subject of several studies. Chau and colleagues identified four factors predicting a poor prognosis in patients receiving first-line treatment for locally advanced or metastatic oesophago-gastric cancer (ECOG PS $\geq 2$, liver metastases, peritoneal metastases, and alkaline phosphatase $\geq 100 \mathrm{U} / \mathrm{L}$ ), and combined them into a prognostic index with distinct survival rates among the different risk groups [15]. Similarly, Lee and colleagues built a clinical model including six factors that predicted a poor prognosis for patients receiving first-line chemotherapy (no previous gastrectomy, albumin $<3.6 \mathrm{~g} / \mathrm{dl}$, alkaline phosphatase $>85 \mathrm{U} / \mathrm{L}$, ECOG PS $\geq 2$, presence of bone metastases, and presence of ascites) that defined three prognosis groups [16]. In second-line therapy, Catalano and colleagues identified five factors that were independently associated with poor overall survival in multivariate analysis: ECOG PS 2, haemoglobin $\leq 11.5 \mathrm{~g} / \mathrm{dl}$, CEA level $>50 \mathrm{ng} / \mathrm{ml}$, the presence of $\geq 3$ metastatic sites of disease, and time to progression (TTP) at first-line chemotherapy $\leq 6$ months [17]. Accordingly, Kanagavel and colleagues identified three independent factors predicting a good prognosis: ECOG PS 0-1, haemoglobin level $\geq 10 \mathrm{~g} / \mathrm{dl}$, and TTP at first-line therapy $\geq 5$ months [18]. Our analysis was based on the individual data for 868 patients treated with second-line chemotherapy, and it identified four independent prognostic factors in multivariate analyses: ECOG PS, LDH level, neutrophils/lymphocytes ratio, and PFS achieved in first-line therapy (Table 4). Our study underlines the importance of ECOG PS when receiving second-line treatment (see Online Resource 1 and Online Resource 2 in the Electronic supplementary material, ESM). After first-line chemotherapy, the patient's general condition may quickly worsen, and a further line of therapy should be chosen after careful assessment of the balance between efficacy and potential toxicity.

A high serum LDH level is associated with poor survival in cases with a solid tumour, where the tumour could be of several types. The rationale behind this is the different metabolism of cancer compared with that of normal cells. Indeed, in neoplastic cells, metabolism favours aerobic glycolysis with the production of lactate associated with an increased uptake of glucose; LDH is the enzyme that reduces pyruvate to lactate, and its level is often raised in aggressive cancer and hematologic malignancies [19]. Our study confirms this evidence, showing that a LDH level $\leq 480 \mathrm{UI} / \mathrm{L}$ is associated with better PFS and OS in the second-line therapy, when the tumour burden may be particularly high (Online Resource 3 and Online Resource 4 in the ESM). Therefore, LDH might be a potential useful and inexpensive prognostic biomarker in AGC.

Recently, the neutrophils/lymphocytes ratio has emerged as a novel prognostic indicator for several cancers [20], given that inflammation may play a pivotal role in disease progression. The results of our analysis are in line with data in the literature, which show that a high neutrophils/lymphocytes ratio is associated with an adverse outcome in many solid tumours. Indeed, in our study, patients with a neutrophils/lymphocytes ratio $<2.7$ seemed to have a better outcome (Online Resource 5 and Online Resource 6 in the ESM).

Finally, in line with previous reports [17, 18, 21], a longer PFS in the first-line therapy ( $\geq 6.8$ months) was a predictor of good prognosis in our series (Figs. 1, 2). This may suggest that patients with rapidly progressing disease represent an unfavourable subset, with decreased chances of benefitting from second-line chemotherapy. As many new agents for the treatment of AGC are emerging [22], enrolment in clinical trials should also be considered as a valuable option in these patients after first progression.

In our report, we used these four independent prognostic factors to define three different risk groups of patients: good, intermediate, and poor risk groups, with a median second-line OS of 7.7 months (95\% CI 6.9-8.7 months), 4.5 months (95\% CI 3.7-5.2 months), and 2.0 months (95\% CI 1.5-2.4), respectively (log-rank $<0.0001$ ); see Fig. 3. Our simple prognostic index clearly identified three distinct risk groups and, if prospectively validated, it could be used for risk stratification in future phase III trials and to inform clinical decision-making. It might help to identify a group of patients that benefit most from a second-line chemotherapy, and clinicians to discuss the prognosis for a patient with that patient. The high number of patients included in our real-life experience-one of the largest Western series ever presented-is most certainly a strength of this work. Currently available second-line treatments such as irinotecan or taxanes were used for the majority of

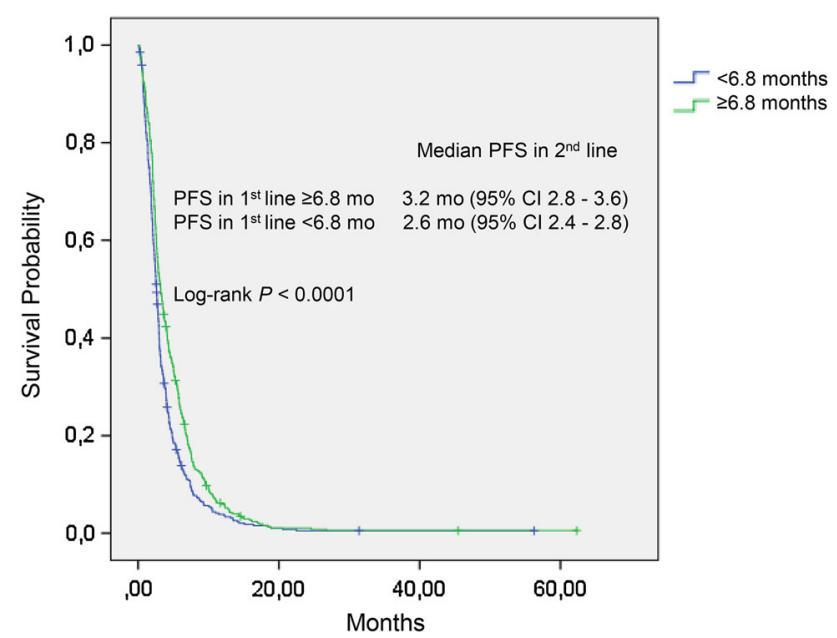

Fig. 1 Survival curves for progression-free survival in second-line therapy according to the progression-free survival achieved in the first-line therapy. PFS progression-free survival, $C I$ confidence interval, mo months 


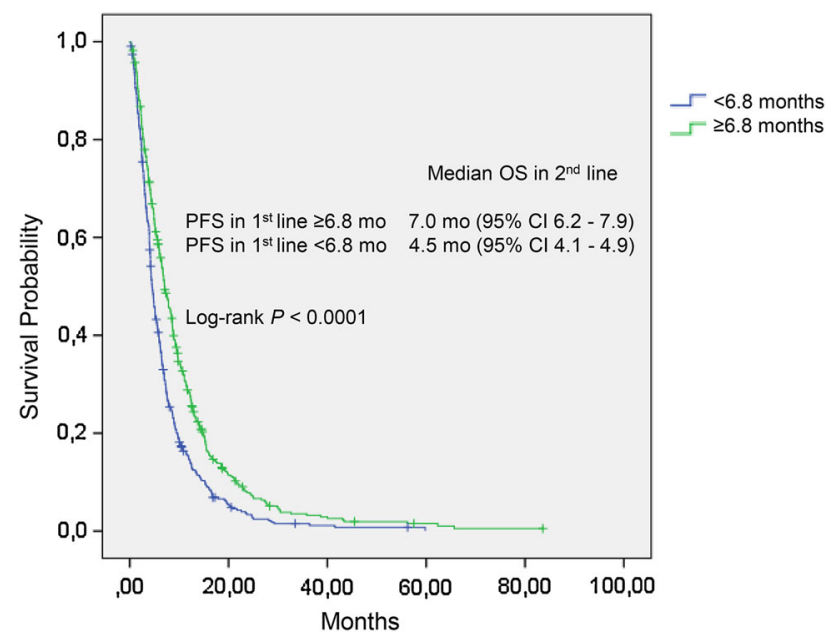

Fig. 2 Survival curves for overall survival in second-line therapy according to the progression-free survival achieved in the first-line therapy. PFS progression-free survival, $C I$ confidential interval, $O S$ overall survival, mo months

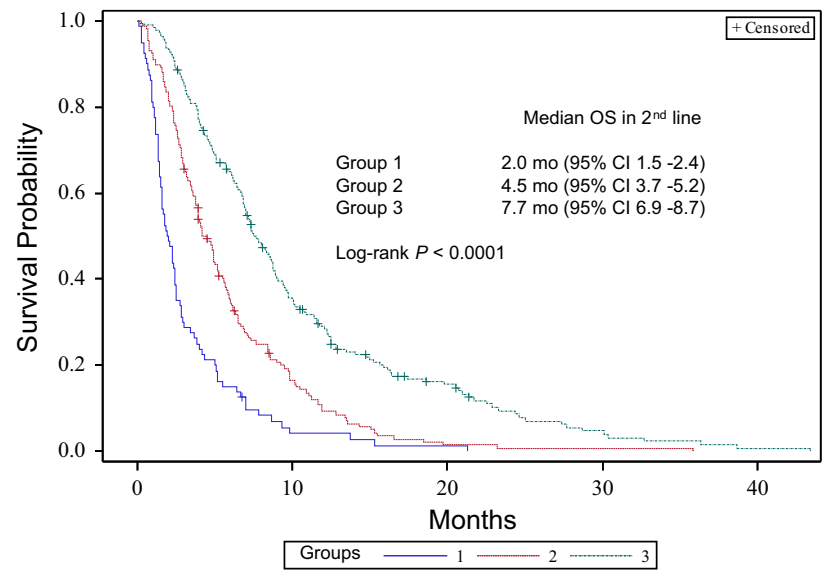

Fig. 3 Survival curves for overall survival in second-line therapy according to risk group. $C I$ confidence interval, $O S$ overall survival, mo months

the patients included, which is representative of routine practice; moreover, the patients were included consecutively with no major selection bias (i.e. PFS and OS in the first- and second-line therapies were as expected from randomised trials and previous case series). However, the patients included in our analysis represent a selected population that was able to tolerate a further line of treatment after disease progression.

However, our work also has its weaknesses: it is retrospective in nature, and lacks data on other potentially prognostic factors (CEA, PCR, albumin). Moreover, the vascular endothelial growth factor receptor (VEGFR)-2 inhibitor ramucirumab was prescribed in a minority of patients either as monotherapy or combined with paclitaxel: it would be interesting to confirm the value of our prognostic factors (specifically $\mathrm{LDH}$ ) in ramucirumab- treated AGC patients, as proposed by Lordick et al. [23], to verify the usefulness of these easy-to-collect prognostic variables when deciding whether to start the patient on second-line ramucirumab with systemic chemotherapy or without it. In contrast to other studies, we did not find a significant correlation between haemoglobin level at the start of second-line therapy and survival. The higher median haemoglobin level noted in our patients at the start of second-line therapy (median value of $11.5 \mathrm{~g} / \mathrm{dl}$ ) may have diluted its impact on the final outcome.

\section{Conclusions}

In conclusion, AGC patients with a favourable ECOG PS, lower LDH levels, and a lower neutrophils/lymphocytes ratio at the start of second-line therapy seem to have better outcomes, regardless of age and intensity of treatment received. A longer PFS in the first-line therapy also showed positive prognostic value. The principal advantage of these four parameters is that they are inexpensive to determine and rapidly available to all oncologists in routine clinical practice. Given the lack of a standard second-line therapy, the results of our study might help clinicians to identify the optimal candidate for further treatment after progression to first-line chemotherapy. Moreover, if prospectively confirmed in other studies, the prognostic index we derived from the combination of these easily available parameters could be implemented in future clinical trials of pretreated AGC cases as stratification factors.

Acknowledgements Work in the unit of DM was supported by startup Grant No. 10,129 and Investigator Grant No. 19,111 through the Associazione Italiana per la Ricerca sul Cancro (AIRC), and by Basic Research Project 2015 through the University of Verona.

\section{Compliance with ethical standards}

This article does not contain any studies with human or animal subjects performed by any of the authors. Although this is not a prospective clinical trial but a retrospective study, we obtained approval for the study from our local scientific review board.

Conflict of interest The authors declare that they have no conflict of interest.

\section{References}

1. Global Burden of Disease Cancer Collaboration, Fitzmaurice C, Dicker D, Pain A, Hamavid H, Moradi-Lakeh M, MacIntyre MF, et al. The Global Burden of Cancer 2013. JAMA Oncol. 2015;1(4):505-27.

2. Siegel RL, Miller KD, Jemal A. Cancer statistics, 2016. CA Cancer J Clin. 2016;66(1):7-30.

3. Shah MA. Update on metastatic gastric and esophageal cancers. J Clin Oncol. 2015;33(16):1760-9. 
4. Wagner AD, Grothe W, Haerting J, Kleber G, Grothey A, Fleig WE. Chemotherapy in advanced gastric cancer: a systematic review and meta-analysis based on aggregate data. J Clin Oncol. 2006;24(18):2903-9.

5. Cunningham D, Starling N, Rao S, Iveson T, Nicolson M, Coxon F, et al. Upper Gastrointestinal Clinical Studies Group of the National Cancer Research Institute of the United Kingdom. Capecitabine and oxaliplatin for advanced esophagogastric cancer. N Engl J Med. 2008;358(1):36-46.

6. Van Cutsem E, Moiseyenko VM, Tjulandin S, Majlis A, Constenla M, Boni C, et al. V325 Study Group. Phase III study of docetaxel and cisplatin plus fluorouracil compared with cisplatin and fluorouracil as first-line therapy for advanced gastric cancer: a report of the V325 Study Group. J Clin Oncol. 2006;24(31):4991-7.

7. Bang YJ, Van Cutsem E, Feyereislova A, Chung HC, Shen L, Sawaki A, et al. ToGA Trial Investigators. Trastuzumab in combination with chemotherapy versus chemotherapy alone for treatment of HER2-positive advanced gastric or gastro-oesophageal junction cancer (ToGA): a phase 3, open-label, randomised controlled trial. Lancet. 2010;376(9742):687-97.

8. Kim HS, Kim HJ, Kim SY, Kim TY, Lee KW, Baek SK, et al. Second-line chemotherapy versus supportive cancer treatment in advanced gastric cancer: a meta-analysis. Ann Oncol. 2013;24(11):2850-4.

9. Janowitz T, Thuss-Patience P, Marshall A, Kang JH, Connell C, Cook $\mathrm{N}$, et al. Chemotherapy vs supportive care alone for relapsed gastric, gastroesophageal junction, and oesophageal adenocarcinoma: a meta-analysis of patient-level data. $\mathrm{Br} \mathrm{J}$ Cancer. 2016;114(4):381-7.

10. Koizumi W, Narahara H, Hara T, Takagane A, Akiya T, Takagi $\mathrm{M}$, et al. S-1 plus cisplatin versus S-1 alone for first-line treatment of advanced gastric cancer (SPIRITS trial): a phase III trial. Lancet Oncol. 2008;9(3):215-21.

11. Ford HE, Marshall A, Bridgewater JA, Janowitz T, Coxon FY, Wadsley J, et al. COUGAR-02 Investigators. Docetaxel versus active symptom control for refractory oesophagogastric adenocarcinoma (COUGAR-02): an open-label, phase 3 randomised controlled trial. Lancet Oncol. 2014;15(1):78-86.

12. Hironaka S, Ueda S, Yasui H, Nishina T, Tsuda M, Tsumura T, et al. Randomized, open-label, phase III study comparing irinotecan with paclitaxel in patients with advanced gastric cancer without severe peritoneal metastasis after failure of prior combination chemotherapy using fluoropyrimidine plus platinum: wJOG 4007 trial. J Clin Oncol. 2013;31(35):4438-44.

13. Fuchs CS, Tomasek J, Yong CJ, Dumitru F, Passalacqua R, Goswami C, et al. REGARD Trial Investigators. Ramucirumab monotherapy for previously treated advanced gastric or gastro- oesophageal junction adenocarcinoma (REGARD): an international, randomised, multicentre, placebo-controlled, phase 3 trial. Lancet. 2014;383(9911):31-9.

14. Wilke H, Muro K, Van Cutsem E, Oh SC, Bodoky G, Shimada Y, et al. RAINBOW Study Group. Ramucirumab plus paclitaxel versus placebo plus paclitaxel in patients with previously treated advanced gastric or gastro-oesophageal junction adenocarcinoma (RAINBOW): a double-blind, randomised phase 3 trial. Lancet Oncol. 2014;15(11):1224-35.

15. Chau I, Norman AR, Cunningham D, Waters JS, Oates J, Ross PJ. Multivariate prognostic factor analysis in locally advanced and metastatic esophago-gastric cancer-pooled analysis from three multicenter, randomized, controlled trials using individual patient data. J Clin Oncol. 2004;22(12):2395-403.

16. Lee J, Lim T, Uhm JE, Park KW, Park SH, Lee SC, et al. Prognostic model to predict survival following first-line chemotherapy in patients with metastatic gastric adenocarcinoma. Ann Oncol. 2007;18(5):886-91.

17. Catalano V, Graziano F, Santini D, D'Emidio S, Baldelli AM, Rossi D, et al. Second-line chemotherapy for patients with advanced gastric cancer: who may benefit? $\mathrm{Br} \mathrm{J}$ Cancer. 2008;99(9):1402-7.

18. Kanagavel D, Pokataev IA, Fedyanin MY, Tryakin AA, Bazin IS, Narimanov MN, et al. A prognostic model in patients treated for metastatic gastric cancer with second-line chemotherapy. Ann Oncol. 2010;21(9):1779-85.

19. Silvestris N, Scartozzi M, Graziano G, Santini D, Lorusso V, Maiello E, et al. Basal and bevacizumab-based therapy-induced changes of lactate dehydrogenases and fibrinogen levels and clinical outcome of previously untreated metastatic colorectal cancer patients: a multicentric retrospective analysis. Expert Opin Biol Ther. 2015;15(2):155-62.

20. Templeton AJ, McNamara MG, Šeruga B, Vera-Badillo FE, Aneja P, Ocaña A, et al. Prognostic role of neutrophil-to-lymphocyte ratio in solid tumors: a systematic review and metaanalysis. J Natl Cancer Inst. 2014;106(6):dju124.

21. Hashimoto K, Takashima A, Nagashima K, Okazaki SS, Nakajima TE, Kato K, et al. Progression-free survival in first-line chemotherapy is a prognostic factor in second-line chemotherapy in patients with advanced gastric cancer. J Cancer Res Clin Oncol. 2010;136(7):1059-64.

22. Aprile G, Giampieri R, Bonotto M, Bittoni A, Ongaro E, Cardellino GG, et al. The challenge of targeted therapies for gastric cancer patients: the beginning of a long journey. Expert Opin Investig Drugs. 2014;23(7):925-42.

23. Lordick F, Janjigian YY. Clinical impact of tumour biology in the management of gastroesophageal cancer. Nat Rev Clin Oncol. 2016;13(6):348-60. 